Planetary Systems in the Universe - Observation, Formation and Evolution

Proceedings IAU Symposium No. 202, (C2004 IAU

Alan Penny, Pawel Artymowicz, Anne-Marie Lagrange, 8 Sara Russell, eds.

\title{
Direct Detection of Thermal Emission from Extra-Solar Planets
}

\author{
Matthew Kenworthy, Philip Hinz and Roger Angel \\ Steward Observatory, University of Arizona, 933 N. Cherry Avenue, \\ Tucson AZ 85721, USA
}

\begin{abstract}
We present a new test for the direct detection of extra-solar planets that relies on the presence of a strong molecular absorption band in the thermal emission from the planetary atmosphere. This signature will be sought by the method of differential astrometry, in which the astrometric centroid positions are compared in and out of the molecular band.
\end{abstract}

\section{Introduction}

Since the discovery of hot Jupiters via Doppler techniques, many other detection strategies have been explored that exploit the proximity of the planet to its parent star, many of which are discussed at this symposium. We propose an extension of the method of differential spectro-astrometry to detect these hot Jupiters.

Spectro-astrometry is the measurement of the relative displacement of a stellar system as a function of wavelength. In the case of a binary system, emission or absorption features present in one object but not the other cause an astrometric displacement, the size of which gives an estimate of the minimum separation of the two components.

Jeremy Bailey has presented results from the AAT where he reports detection of line features with accuracies of 5 milliarcseconds (mas) from a spectrum in 2 arcsecond seeing (Bailey 1998). Since the method relies on determining the position of the centroid of the imaged system, shifts much smaller than the diffraction limit can be detected when the star image is first corrected to the diffraction limit by $\mathrm{AO}$.

\section{The Spectra of Hot Jupiters}

Since the discovery of Jupiter mass planets that lie within $0.1 \mathrm{AU}$ of their parent star, theorists have been working on models of the spectral emission from the planet. The peak of the thermal $(T \sim 1600 K)$ emission is in the near infra-red typically $1-2 \mu \mathrm{m}$, where the contrast between star and planet is considerably better than that in the visible spectrum.

The spectrum shows broad molecular absorption from water vapour and methane at 1.13 and $1.40 \mu \mathrm{m}$. Modelling done by Sudarsky and Burrows (2000) and Sudarsky et al. (2000) of the planet around $\tau$ Boo shows deep absorption either side of thermal emission centered at $1.27 \mu \mathrm{m}$ (see Figure 1). 




Figure 1. Theoretical flux ratio of $\tau$ Boo and its planet versus wavelength adapted from Sudarsky \& Burrows (2000). The spectrum has been smoothed to represent the flux as seen at a spectral resolution $R \sim 25(0.05 \mu \mathrm{m})$. The greatest contrast is seen around $1.27 \mu \mathrm{m}$.

An estimate can be made of the expected astrometric signal for the planet around $\tau$ Boo. The planet has a semi-major axis $a=0.042 A U$, the distance $d=15 p c$ and the $\mathrm{V}$-band magnitude $V=4.50 \mathrm{mag}$ (Butler et al. 1997). The greatest elongation of the planet is therefore $0.042 / 15=2.8 \mathrm{mas}$.

From Figure 2 the expected contrast ratio around $1.27 \mu \mathrm{m}$ is $5.0 \times 10^{-5}$ and $1.0 \times 10^{-5}$ in the absorption bands. This leads to an on-band signal of $2.8 \times 10^{-3} / 5 \times 10^{-5}=0.14 \mu a s$ and off-band the signal is $2.8 \times 10^{-3} /\left(5 \times 10^{-5}\right.$ $\left.1.0 \times 10^{-5}\right)=0.035 \mu a s$ leading to a shift of $0.10 \mu a s$.

\section{Spectro-astrometry with the LBTI}

A sketch of the LBTI instrument is given in Figure 2. When the dispersion axis of the prism is perpendicular to the line connecting $\tau$ Boo and its planet, there will be a $0.1 \mu$ as kink in the dispersed spectrum. As the night progresses, the telescope baseline changes with respect to the sky and so the signal will vary in strength. A K-prism allows a rotation of 180 degrees of the spectral image, 


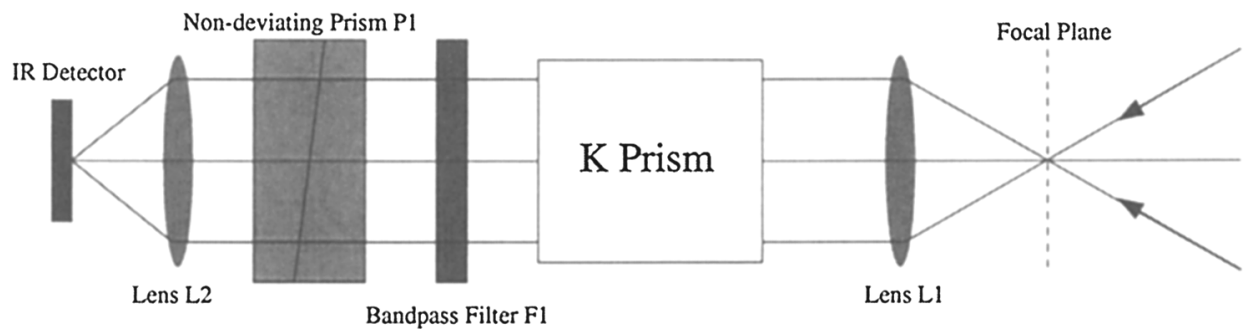

Figure 2. A sketch of the proposed instrument. Lens L1 collimates the beam, a $\mathrm{J}$ band filter $\mathrm{F} 1$ selects the two absorption band regions either side of the peak at $1.27 \mu \mathrm{m}$ and the non-deviating prism introduces a low amount of dispersion along the long axis of the LBTI PSF fringes. The dispersion is chosen such that the length of the spectrum is approximately 6 times the diameter of the Airy disk of a single $8.4 \mathrm{~m}$ telescope, leading to $R \sim 25$.

averaging any other sources of random noise, whilst the planets signal increases as more frames are processed.

The sensitivity of the detector is dominated by photon shot noise - for a given number of photons $N$ within a given fringe of the PSF, the r.m.s accuracy to which its centroid can be calculated is the FWHM of the fringe divided by $\sqrt{ } N$. In order to detect the signal of the $\tau$ Boo planet with a $5 \sigma$ limit, the number of photons needed is $(9.1 \mathrm{mas} / 0.02 \mu a s)^{2}=2 \times 10^{11}$ photons per spectral resolving element.

Taking the efficiency of the telescope optics and instrument to be $50 \%$, the AO system producing a Strehl ratio of $50 \%$ and that only photons within the first Airy ring are to be considered, this gives a total on-sky integration time of 4.3 minutes.

The actual length of time will be longer given multiple readouts and duty cycle time, but this is well within the reach of the LBTI. A similar calculation for the MMT with the AO system gives 6.4 hours, making it possible to get a 3 sigma detection with one night, including overheads.

Acknowledgments. MAK is indebted to David Sudarsky for providing data from his model atmospheres at short notice.

\section{References}

Bailey, J. 1998, MNRAS, 301, 161

Butler, R., Marcy, G., Williams, E., Hauser, H., Shirts, P. 1997, ApJ, 474, L115

Sudarsky, D., Burrows, A., Pinto, P. 2000, ApJ, 538, 885

Sudarsky, D., Burrows, A. 2000, in preparation 